\title{
The comparative development of elevated resistance to macrolides in community-acquired pneumonia caused by Streptococcus pneumoniae
}

This article was published in the following Dove Press journal:

Drug Design, Development and Therapy

3 October 2014

Number of times this article has been viewed

\section{Josef Yayan}

Department of Internal Medicine, Division of Pulmonary, Allergy and Sleep Medicine, Saarland University Medical Center, Homburg/Saar, Germany
Correspondence: Josef Yayan Department of Internal Medicine, Division of Pulmonary, Allergy and Sleep Medicine, Saarland University Medical

Center, Kirrberger Straße, D-6642I

Homburg/Saar, Germany

Tel +49 684I I6 21620

Fax +49 684I 1623602

Email josef.yayan@hotmail.com
Background: Community-acquired pneumonia (CAP) is an acute inflammation of the lungs, which is often caused by Streptococcus pneumoniae. CAP is the leading cause of death by infectious disease in industrialized countries. Therefore, an immediate and effective antibiotic therapy is of great importance for the nonfatal outcome of the disease. The literature contains increasing data about the development of resistance to antibiotics that are used for the treatment of CAP caused by S. pneumoniae; this article also examines the possible development of resistance to antibiotics in $S$. pneumoniae in recent years.

Methods: Within the study period of 2004-2014, all hospital charts from patients with CAP caused by $S$. pneumoniae were collected from the Department of Internal Medicine, Saarland University Medical Center, Homburg/Saar, Germany. The tracheal secretions of S. pneumoniae in CAP patients were obtained by bronchoalveolar lavage; bronchial aspirates were obtained through flexible bronchoscopy and directly from sputum, and blood cultures were examined microbiologically for microorganisms.

Results: From a total of 100 patients with CAP caused by S. pneumoniae, 23 (53.49\% [34.78\% female], 95\% confidence interval, 38.58-68.4) patients with a mean age of 59.78 \pm 15.77 years met the inclusion criteria of this investigation. These patients were compared to a total of $20(46.51 \%$ [35\% female], 95\% confidence interval, 31.6-61.42) patients with a mean age of 58.9 \pm 13.36 years with CAP who were infested with $S$. pneumoniae. In the latter group, the streptococcal antigen was detected in pulmonary aspirations by bronchoscopy or in urine using polymerase chain reaction and a rapid pneumococcal test. Penicillin G and vancomycin had a high rate of sensitivity on the antibiogram for $S$. pneumoniae, which was obtained by bronchoalveolar lavage, bronchial aspirates through flexible bronchoscopy, and directly from sputum. Even though the rates obtained were without statistical significance, S. pneumoniae had a high resistance to macrolides, namely erythromycin, in patients with CAP. Macrolides, specifically erythromycin (17.39\%) and azithromycin (4.35\%) and other classes of antibiotics such as tetracycline (4.35\%), had a statistically significant resistance to streptococcal pneumonia in patients with $\operatorname{CAP}(P=0.0009)$.

Conclusion: Increased resistance was found for macrolides and tetracycline in patients with CAP by S. pneumoniae.

Keywords: drug-resistant Streptococcus pneumoniae, drug-resistant antibiotics, antimicrobial therapy, pneumococcal pneumonia, penicillin resistance

\section{Introduction}

Community-acquired pneumonia (CAP) is defined as an acute lower respiratory tract infection. ${ }^{1}$ Various pathogens can cause this acute infectious disease of the lungs, but it is mainly caused by Streptococcus pneumoniae. ${ }^{1}$ 
Many millions of people worldwide are annually affected by CAP. ${ }^{2} \mathrm{CAP}$ has a high mortality rate, so it is important to detect high-risk patients who are vulnerable to complications and death. Hospital-acquired pneumonia has a mortality rate of up to $50 \%$. ${ }^{2}$ Pneumonia is classified upon a causing pathogen, or it is called after how pneumonia is acquired. ${ }^{2}$

Patients with CAP often require intensive therapeutic measures such as intensive care and mechanical ventilation. ${ }^{3}$ The management of streptococcal-related pneumonia is made more difficult by the appearance of several antimicrobial or resistant germs. ${ }^{4,5}$ Therefore, the successful treatment of drug-resistant pneumococcal pneumonia represents a challenge for physicians. There is an association between the usage of antiseptic agents and the occurrence of resistance in S. pneumoniae. ${ }^{6}$ Resistance to penicillin and other beta-lactamase inhibitors has become prevalent, and there is increasing confirmation that resistance to macrolides can lead to a reduced clinical reaction. Fluoroquinolones have been broadly used to treat respiratory tract infections, among others, and pneumococcal resistance to these agents is rising, although it presently remains small. ${ }^{6}$ There are reports that resistance to fluoroquinolones can develop during treatment and may be reflected in a lack of clinical response. ${ }^{5}$ Several clinical and epidemiological variables can be useful to identify patients who are at risk of infections with antibiotic-resistant pneumococci, and these at-risk patients are those who would benefit the most from a pneumococcal vaccination program. ${ }^{6}$

The present investigation was conducted to identify drug-resistant $S$. pneumoniae after microbial cultures were identified from the blood, bronchoalveolar lavage or bronchial aspirates, or the sputum of the patients studied. To this aim, we collected information for all patients with S. pneumoniae (according to the International Classification of Diseases) who were examined for microbiome from the hospital database at the Department of Internal Medicine, Saarland University Medical Center, Germany.

The aim of this study is to determine the resistance situation of antibiotics used to treat $S$. pneumoniae in order to make the right initial selection of antibiotic therapy for future patients suffering from CAP caused by S. pneumoniae.

\section{Materials and methods}

\section{Patients}

This quality control study retrospectively examined the resistance profile in the antibiogram of patients with CAP caused by S. pneumoniae from January 1, 2004 to June 23, 2014. The study population consisted of patients with CAP caused by S. pneumoniae detected after microbial isolation using blood culture, bronchoalveolar lavage, and pulmonary aspiration by bronchoscopy. These patients were compared to a group of patients diagnosed with CAP by $S$. pneumoniae detected by a polymerase chain reaction (PCR) of pulmonary aspiration by bronchoscopy or urine and a rapid pneumonia test.

Pneumococcal pneumonia has been defined as an acute inflammatory disease of the respiratory tract caused by S. pneumoniae that affects the alveolar and interstitium spaces. Pneumococci are the most common pathogens in CAP. CAP symptoms are classified as sudden onset with shaking and high fever; cough; shortness of breath; chest pain while breathing, possibly caused by concomitant pleurisy; and any production of sputum. The diagnosis of CAP caused by $S$. pneumoniae was made under the main criterion of newonset pulmonary infiltrate in the chest X-ray and by clinical examination, raised respiratory rate, fever over $38.5^{\circ} \mathrm{C}$ or hypothermia less than $36.6^{\circ} \mathrm{C}$, purulent expectoration, bronchial breathing by auscultation of fine bubbling crackles, and testing fremitus positive. The classification of pneumococcal pneumonia was performed in each case according to the latest edition of the International Classification of Diseases (ICD J13) from 2004 to 2014. Following the diagnosis of CAP due to $S$. pneumoniae, all adults over 18 years of age were included in this study; all study patients were subjected to the following investigations upon admission: chest radiograph; full blood count; urea, electrolytes, and liver function tests; C-reactive protein (CRP); and oxygenation assessment.

The indication for the microbiological examination was performed either explicitly because of suspected respiratory infection or as routine. Microbiological testing was explicitly performed for pathogenic microbes, viruses, fungi, legionellae, mycobacteria, nocardiosis, actinomycetales, pneumocystis jiroveci (carinii), and PCR (eg, atypical pneumonia pathogens and mycobacteria).

Several blood cultures were employed to detect pathogens that propagate through the bloodstream. Following careful alcohol skin disinfection (74.1\% ethanol and propan-2-ol $10 \%$ ) by Softasept ${ }^{\circledR}$ N (B. Braun Melsungen PLC, Melsungen, Germany), a minimum of $20 \mathrm{~mL}$ of blood in Braun Injekt ${ }^{\circledR}$ single-use syringes (B. Braun Melsungen PLC) was taken through venipuncture with a blood collection needle (Safety-Multifly ${ }^{\circledR}$; Sarstedt AG \& Co., Nümbrecht, Germany) and injected into two specific media - BACTEC Plus Aerobic/F and Plus Anaerobic/F media (BD, Becton, Dickinson and Company, Heidelberg, Germany) and enriched soybean-casein digest broth media. After injecting the blood culture bottles with new needles, they were sent to the microbiology department, where they were entered 
into a blood culture machine that incubated the specimens at body temperature. The blood culture instrument reported positive blood cultures with bacteria present; most cultures were monitored for 5 days, after which negative vials were removed. If a vial was positive, a microbiologist performed a Gram stain on the blood for a rapid general identification of the bacteria, which they reported to the attending physician of the bacteremia patient. The blood was then cultured onto agar plates to isolate the pathogenic organism for culture and susceptibility testing, which takes up to 3 days. This culture and sensitivity process identified the species of bacteria. Antibiotic sensitivities were then assessed on the bacterial isolate to inform clinicians about appropriate antibiotics for treatment.

The minimal inhibitory concentration was characterized as a value expressed in $\mathrm{mg} / \mathrm{L}$ for the effectiveness of an antibiotic as well as for the assessment of the resistance of a pathogen according the International Organization for Standardization Standard 20776-1. ${ }^{6}$ The clinical interpretation of the results was performed using minimal inhibitory concentration breakpoints for penicillin $\mathrm{G}$ in the categories of sensitive $\leq 0.06 \mathrm{mg} / \mathrm{L}$, intermediate $=0.12-1 \mathrm{mg} / \mathrm{L}$, and resistant $>1 \mathrm{mg} / \mathrm{L}$.

There were also some attempts to use molecular biological methods, usually the PCR for the detection of S. pneumoniae in bronchial secretions and in urine. For this examination, urine was collected in a $10 \mathrm{~mL}$ Urine-Monovette ${ }^{\circledR}$ (Sarstedt AG \& Co.), which was also used for the rapid urinary pneumococcal antigen testing on an immunochromatographic membrane assay.

The secretion from the mouth-nasal cavity and trachea was obtained differently depending on the particular case; the commonly used methods applied were bronchoalveolar lavage, pulmonary aspiration, throat swabs, and sputum samples. The bronchoalveolar lavage was applied in the context of a bronchoscopy; in this case, about $20 \mathrm{~mL}$ of $0.9 \%$ saline solution was administered under local anesthesia and aspirated through the fiber optic bronchoscope. The aspirate thus obtained was deposited into three different sterile specimen traps (tracheal suction set; Dahlhausen Medizintechnik, Köln, Germany). Pulmonary aspirations were also collected by fiber optic bronchoscopy through aspiration into the same sterile specimen traps described above. The throat swab was collected with a commercial cotton swab transport system (Copan Italia, Brescia, Italy) by rotating the swab with slight pressure on the palatal arch of patients with CAP. Recovery of sputum was performed by expectoration into a $30 \mathrm{~mL}$ sterile sputum collection tube (Salivette ${ }^{\circledR}$; Sarstedt AG \& Co.) and sent to the laboratory for analysis. The inclusion criteria for this study were all patients with CAP whose tracheal secretions were detected microbiologically for $S$. pneumoniae. All patients with pneumonia who were not subjected to a microbiological test were excluded from this study.

The quantitative determination of CRP in human serum and plasma was measured after sample collection in a $4.7 \mathrm{~mL}$ lithium heparin Sarstedt AG \& Co. Monovette ${ }^{\circledR}$ (orange top) using a standard immunoturbidimetric assay on the Roche COBAS $^{\circledR}$ INTEGRA system c 701 (Roche diagnostics Ltd, Mannheim, Germany) (the normal value of which is less than $6 \mathrm{mg} / \mathrm{L}$ ). The determination of the leukocyte count (normal range $4,000-10,000 / \mu \mathrm{L})$ in the blood was carried out as a routine part of blood counts after collection in a $2.7 \mathrm{~mL}$ ethylenediaminetetraacetic acid (EDTA) Monovette ${ }^{\circledR}$ by flow cytometry.

We analyzed the acute and chronic comorbidities of drug sensitivity and resistance and the length of hospital stay between the study population groups. Due to the retrospective nature of the study protocol, the Medical Association of Saarland's Institutional Review Board waived the need for informed consent.

\section{Statistical analysis}

The data were expressed in proportion, mean, and standard deviation wherever appropriate. We calculated 95\% confidence intervals (CIs) for the total number of patients with CAP by $S$. pneumoniae. A calculation of chi-squared test for two independent standard normal variables of two probabilities was used to compare the association between different antibiotics and S. pneumoniae in antibiograms, comorbidities of drug sensitivity and resistance, blood culture, bronchoalveolar lavage, pulmonary aspiration, and sputum. A calculation of chi-squared test for three independent standard normal variables of two probabilities was used to compare the association between comorbidities, antibiotics, and pneumococcal antigen detection by PCR in pulmonary aspiration and in urine, and rapid pneumococcal test. A one-way analysis of variance for two independent samples was performed to compare CRP, leukocyte counts, and length of hospital stay in drug sensitivity and resistance groups of antibiotics for S. pneumoniae. A one-way analysis of variance for three independent samples was performed to compare mean age, CRP, leukocyte counts, and length of hospital stay, pneumococcal antigens diagnosed by PCR in pulmonary aspiration by bronchoscopy, PCR in urine, and rapid pneumococcal test. Mann-Whitney test statistics were used to compare minimal inhibitory concentration of penicillin $\mathrm{G}$ between sensitivity and resistance groups of 
antibiotics for $S$. pneumoniae. All tests were expressed as two-tailed, and a $P$-value of $<0.05$ was considered to be statistically significant.

\section{Results}

In the hospital database, we found 100 patients with CAP caused by $S$. pneumoniae who had been treated at the Department of Internal Medicine, University Hospital of Saarland, Germany, from 2004 to 2014. A total of 43 patients with CAP by $S$. pneumoniae met the inclusion criteria for this trial. A total of 23 (53.49\% [34.78\% female], 95\% CI, 38.58-68.4) patients with mean age of $59.78 \pm 15.77$ years with CAP were infested with $S$. pneumoniae, which was microbiologically detected by a blood culture or tracheal secretion. A total of 20 (46.51\% [35\% female], 95\% CI, 31.6-61.42) patients with a mean age of $58.9 \pm 13.36$ years with CAP were infested with S. pneumoniae, detected by the streptococcal antigen in their pulmonary aspiration by bronchoscopy or urine using PCR and rapid pneumococcal test.

A high sensitivity rate to penicillin $G$ and vancomycin was identified on the antibiogram after the microbiological culture was taken, but this was without statistical significance (Table 1). The highest resistance profile on the antibiogram was found for macrolides erythromycin, but this was also without statistical significance (Table 1). Specifically, azithromycin had no sensitivity and low resistance to $S$. pneumoniae in the antibiogram with a statistical significance $(P=0.0009)$. Tetracycline also had a high resistance in the antibiogram for $S$. pneumoniae $(P=0.0009)$.

No statistical significance was found between drug sensitivity and resistance groups in the diagnostic tools for identification of pathogens, the inflammatory markers in the blood laboratory, and the minimal inhibitor concentration of antibiotics (Table 2).

Table I Comparison of Streptococcus pneumoniae sensitivity and resistance to drug groups as detected on an antibiogram after microbiology culture

\begin{tabular}{|c|c|c|c|c|}
\hline \multirow[t]{2}{*}{ Drug groups } & \multirow[t]{2}{*}{ Active substance (\%) } & \multicolumn{2}{|l|}{$\mathbf{N}=\mathbf{2 3}$} & \multirow[t]{2}{*}{$P$-value } \\
\hline & & Sensible (\%) & Resistant (\%) & \\
\hline \multirow[t]{3}{*}{ Penicillins } & Penicillin G & 21 (9l.30) & I (4.35) & 0.3011 \\
\hline & Ampicillin & $3(13.04)$ & I (4.35) & 0.1579 \\
\hline & Oxacillin & $2(8.70)$ & 0 & 0.7581 \\
\hline \multirow[t]{3}{*}{ Penicillin + Beta-lactamase inhibitors } & Amoxicillin + Clavulanic acid & I (4.35) & 0 & 0.8274 \\
\hline & Ampicillin + Amoxicillin & I (4.35) & 0 & 0.8274 \\
\hline & Ampicillin + Sulbactam & I (4.35) & 0 & 0.8274 \\
\hline \multirow[t]{3}{*}{ Cephalosporins } & Cefazolin & I (4.35) & 0 & 0.8274 \\
\hline & Cefotaxime & $14(60.87)$ & 0 & 0.4185 \\
\hline & Cefuroxime & $5(21.74)$ & 0 & 0.6271 \\
\hline \multirow[t]{2}{*}{ Tetracycline } & Doxycycline & $17(73.91)$ & 0 & 0.3732 \\
\hline & Tetracycline & 0 & I (4.35) & 0.0009 \\
\hline \multirow[t]{3}{*}{ Macrolides } & Azithromycin & 0 & I (4.35) & 0.0009 \\
\hline & Clarithromycin & I (4.35) & 0 & 0.8274 \\
\hline & Erythromycin & $18(78.26)$ & $4(17.39)$ & 0.1541 \\
\hline Lincosamide & Clindamycin & $16(69.57)$ & I (4.35) & 0.8511 \\
\hline Aminoglycosides & Gentamicin & $4(17.39)$ & I (4.35) & 0.2336 \\
\hline \multirow[t]{3}{*}{ Gyrase inhibitors } & Ciprofloxacin & I (4.35) & 0 & 0.8274 \\
\hline & Levofloxacin & $17(73.91)$ & I (4.35) & $0.884 I$ \\
\hline & Moxifloxacin & $12(52.17)$ & 0 & 0.4535 \\
\hline Nitrofurans & Nitrofurantoin & $\mathrm{I}(4.35)$ & 0 & 0.8274 \\
\hline Carbapenem & Imipenem & I (4.35) & 0 & 0.8274 \\
\hline \multirow[t]{2}{*}{ Glycopeptides } & Teicoplanin & I (4.35) & 0 & 0.8274 \\
\hline & Vancomycin & $22(95.65)$ & 0 & 0.3117 \\
\hline Lipopeptides & Daptomycin & I (4.35) & 0 & 0.8274 \\
\hline Oxazolidinones & Linezolid & $18(78.26)$ & 0 & 0.3596 \\
\hline Trimethoprim + Sulfonamide & Co-trimazole & $12(52.17)$ & $3(13.04)$ & 0.1372 \\
\hline Fenicoles & Chloramphenicol & $14(60.87)$ & I (4.35) & 0.7794 \\
\hline Streptogramins & Quinupristin/Dalfopristin & 17 (73.91) & 0 & 0.3732 \\
\hline Rifampicin & Rifampicin & $5(21.74)$ & 0 & 0.6271 \\
\hline Other & Fosfomycin & $2(8.70)$ & 0 & 0.7581 \\
\hline
\end{tabular}

Note: Significant $P$-values are shown in bold. 
Table 2 Comparison of drug sensitivity and resistance groups in the diagnostic tools for identification of pathogens, the inflammatory markers in the blood laboratory, and the minimal inhibitor concentration of antibiotics

\begin{tabular}{|c|c|c|c|}
\hline & $\begin{array}{l}\text { Drug sensitivity } \\
(n=16)(\%)\end{array}$ & $\begin{array}{l}\text { Drug resistance } \\
(n=7)(\%)\end{array}$ & $P$-value \\
\hline Male & II (68.75) & $3(42.86)$ & 0.2417 \\
\hline Female & $5(31.25)$ & $4(57.14)$ & 0.2417 \\
\hline $\mathrm{CRP}(<6 \mathrm{mg} / \mathrm{L})$ mean $\pm \mathrm{SD}$ & $194.37 \pm|4| .66$ & $104.81 \pm 99.46$ & 0.095350 \\
\hline Leukocyte count $(4,000-10,000 / \mu \mathrm{L})$ mean $\pm \mathrm{SD}$ & $|4| 28.57 \pm 3,,|98.8|$ & $13,244.38 \pm 8,855.44$ & 0.808874 \\
\hline MIC (sensitivity $\leq 0.06 \mathrm{mg} / \mathrm{L}$ ) & 0.012 & 0.016 & $0.3|73|$ \\
\hline Blood culture & $10(62.5)$ & $3(42.86)$ & 0.3819 \\
\hline Bronchoalveolar lavage & 0 & I (I4.29) & 0.1221 \\
\hline Pulmonary aspiration & $4(25)$ & $2(28.57)$ & 0.8576 \\
\hline Sputum & $2(12.5)$ & I (I4.29) & $>0.9999$ \\
\hline Length of hospital stay in days mean $\pm S D$ & $54.14 \pm 19.17$ & $62.25 \pm 14.59$ & 0.276180 \\
\hline
\end{tabular}

Abbreviations: CRP, C-reactive protein; MIC, minimal inhibitory concentration for penicillin G; SD, standard deviation.

According to the antibiogram, the comorbidities did not show statistical significance in relation to sensitivity and resistance of S. pneumoniae to antibiotics (Table 3). Based on PCR for the pneumococcal antigen in pulmonary aspiration compared to urine and the rapid pneumococcal test, no statistical difference was detected between the sexes, inflammatory markers in blood laboratory, and length of hospital stay (Table 4). In addition, no statistical difference was detected after a comparison of comorbidities in a PCR of pulmonary aspiration by bronchoscopy and in urine, and rapid pneumococcal test of identification of pneumococcal antigens (Table 5).

The antibiotic most often used was piperacillin and tazobactam for the treatment of CAP caused by S. pneumoniae over this study period. In development of resistance to streptococcal pneumonia, most patients were switched on to meropenem (Table 6). Further treatment was successful after changing the antibiotic, upon resistance development. None of the study patients were vaccinated against S. pneumoniae.

Although piperacillin and tazobactam were used most often for the treatment of patients with CAP, the comparison of the antibiotics used in these groups showed no statistical difference by means of the PCR of the pneumococcal antigen in bronchial aspirate by bronchoscopy and in urine or by pneumococcal rapid test $(P=0.082198)$.

During the study period, serotype 38 was detected from the blood culture of only one 76-year-old male patient with CAP caused by S. pneumoniae; this serotype was sensitive to all common antibiotics in the antibiogram and showed no antibiotic-associated resistance. Subsequent investigations performed at the National Reference Center for Streptococci diseases (RWTH, Aachen, Germany) showed that it was serotype 38 in this pneumococcal isolate. ${ }^{7,8}$

\section{Discussion}

Although some cases could not be included in this study due to missing values, the number of cases of CAP with $S$. pneumoniae averaged approximately five per year over the last 5 years in this specialized hospital. The reasons for the reduced numbers are varied, and not all of them can be answered in this study. The incidence of the disease could have fallen over the last few years, or other patients may have been treated in other acute hospitals. In any case, the answers are beyond the scope of this report.

The German National Reference Center for Streptococci at the Institute for Medical Microbiology of the University Hospital RWTH, Aachen, Germany, has been conducting observation studies on invasive pneumococcal disease since 1992. ${ }^{7}$ The report from this institution was based on cases of pneumococcal disease in adults, presenting between July 1992 and June 2013, and July 1997 and June 2013, respectively. Among adults for whom conjugate vaccination was not indicated, this percentage diminished from $40 \%-45 \%$ in the 1992-2006 time period to about $8 \%$ in the $2012-2013$ time period. This sensation of falling incidences of pneumococcal disease was meant as herd protection. The resistance rate against penicillin $\mathrm{G}$ persisted at less than $2.5 \%$ among adults during the study period. Since 2005-2006, macrolide resistance levels have declined to about $8 \%$ among adults. The unbroken reduction of vaccine serotypes as well as the falling pneumococcal disease prevalence since the start of childhood vaccinations suggests the triumph of pneumococcal conjugate vaccination. ${ }^{7}$ The rate of resistance to penicillin $\mathrm{G}$ in this study of $4.35 \%$ increased as a percentage but not in number. Overall, a high response rate was observed to penicillin $\mathrm{G}$ in this study. Although no statistical significance was calculated for macrolipids, resistance to $S$. pneumoniae was $21.74 \%$ in this study. 
Table 3 Comparison of comorbidities for drug sensitivity and resistance for Streptococcus pneumoniae in antibiograms

\begin{tabular}{|c|c|c|c|}
\hline & $\begin{array}{l}\text { Drug sensitivity } \\
(n=16)(\%)\end{array}$ & $\begin{array}{l}\text { Drug resistance } \\
(n=7)(\%)\end{array}$ & $P$-value \\
\hline \multicolumn{4}{|l|}{ Cardiovascular disease } \\
\hline Anemia & I (6.25) & 0 & 0.4988 \\
\hline Acute lymphoblastic leukemia & I (6.25) & 0 & 0.4988 \\
\hline Atrial fibrillation & $5(31.25)$ & 0 & 0.0946 \\
\hline Cardiac decompensation & $2(12.5)$ & 0 & 0.3276 \\
\hline Cardiac dysrhythmia & 0 & I (I4.29) & 0.1221 \\
\hline Cardiomyopathy & I (6.25) & 0 & 0.4988 \\
\hline Chronic lymphoblastic leukemia & I (6.25) & 0 & 0.4988 \\
\hline Chronic venous insufficiency & I (6.25) & 0 & 0.4988 \\
\hline Coronary artery disease & $2(12.5)$ & I (I4.29) & 0.9096 \\
\hline Heart failure & I (6.25) & 0 & 0.4988 \\
\hline Hypertension & $3(18.75)$ & $3(42.86)$ & 0.2257 \\
\hline Myocardial infarction & I $(6.25)$ & 0 & 0.4988 \\
\hline Peripheral arterial occlusive disease & $I(6.25)$ & 0 & 0.4988 \\
\hline Sepsis & $\mathrm{I}(6.25)$ & I (I4.29) & 0.5291 \\
\hline Valvular heart disease & $4(28.57)$ & 0 & 0.1455 \\
\hline \multicolumn{4}{|l|}{ Pulmonary disease } \\
\hline Acute respiratory distress syndrome & 0 & I (I4.29) & 0.1221 \\
\hline Alveolar proteinosis & 0 & I (I4.29) & 0.1221 \\
\hline Asthma & $2(12.5)$ & I (I4.29) & 0.9096 \\
\hline Bronchiectasis & 0 & I (I4.29) & 0.1221 \\
\hline Chronic obstructive bronchitis & $4(28.57)$ & $2(28.57)$ & 0.8576 \\
\hline Emphysema & 0 & I (I4.29) & 0.1221 \\
\hline Lung tumor & I (6.25) & 0 & 0.4988 \\
\hline Pleuritis & 0 & I (I4.29) & 0.1221 \\
\hline Pulmonary embolism & I (6.25) & I (14.29) & 0.5291 \\
\hline Respiratory failure & $2(12.5)$ & 3 & 0.1044 \\
\hline Respiratory global insufficiency & 0 & I (I4.29) & 0.1221 \\
\hline State after pulmonary embolism & I (6.25) & 0 & 0.4988 \\
\hline \multicolumn{4}{|l|}{ Gastrointestinal disease } \\
\hline Appendectomy & 0 & I (I4.29) & 0.1221 \\
\hline Cholecystectomy & I (6.25) & 0 & 0.4988 \\
\hline Colitis & 0 & I (I4.29) & 0.1221 \\
\hline Diabetes & $2(12.5)$ & I (I4.29) & 0.9096 \\
\hline Hepatitis C & I (6.25) & 0 & 0.4988 \\
\hline Hyperlipidemia & I (6.25) & 0 & 0.4988 \\
\hline \multicolumn{4}{|l|}{ Kidney disease } \\
\hline Contracted kidney & 0 & I (I4.29) & 0.1221 \\
\hline Cushing's disease & 0 & I (14.29) & 0.1221 \\
\hline \multicolumn{4}{|l|}{ Gynecological disorders } \\
\hline Hysterectomy & I (6.25) & 0 & 0.4988 \\
\hline \multicolumn{4}{|l|}{ Orthopedic disorders } \\
\hline CREST syndrome & I (6.25) & 0 & 0.4988 \\
\hline Fibromyalgia & I $(6.25)$ & 0 & 0.4988 \\
\hline Myelodysplastic syndrome & I (6.25) & 0 & 0.4988 \\
\hline Osteoarthritis & $2(12.5)$ & 0 & 0.3276 \\
\hline Osteoporosis & I (6.25) & 0 & 0.4988 \\
\hline \multicolumn{4}{|l|}{ Nervous system disorders } \\
\hline Brain tumor & 0 & I (I4.29) & 0.1221 \\
\hline Diabetes insipidus & 0 & I (14.29) & 0.1221 \\
\hline Epilepsy & I (6.25) & 0 & 0.4988 \\
\hline Parkinson's disease & I $(6.25)$ & 0 & 0.4988 \\
\hline Pituitary adenoma & 0 & I (I4.29) & 0.1221 \\
\hline Prior stroke & I (6.25) & 0 & 0.4988 \\
\hline
\end{tabular}


Table 3 (Continued)

\begin{tabular}{|c|c|c|c|}
\hline & $\begin{array}{l}\text { Drug sensitivity } \\
(n=16)(\%)\end{array}$ & $\begin{array}{l}\text { Drug resistance } \\
(n=7)(\%)\end{array}$ & $P$-value \\
\hline \multicolumn{4}{|l|}{ Psychiatric disorders } \\
\hline Alcohol intoxication & 0 & I (I4.29) & 0.1221 \\
\hline Depression & 0 & I (I4.29) & 0.1221 \\
\hline Ex-nicotine abuse & I (6.25) & 0 & 0.4988 \\
\hline Nicotine abuse & I (6.25) & I (I4.29) & 0.5291 \\
\hline \multicolumn{4}{|l|}{ Skin disorders } \\
\hline Allergy & $3(18.75)$ & I (I4.29) & 0.7949 \\
\hline Contrast agent allergy & 0 & I (I4.29) & 0.1221 \\
\hline Exsiccosis & 0 & I (I4.29) & 0.1221 \\
\hline Pollinosis & 0 & I (I4.29) & 0.1221 \\
\hline \multicolumn{4}{|l|}{ Ophthalmological disorders } \\
\hline Cataract & $3(18.75)$ & I (I4.29) & 0.7949 \\
\hline Glaucoma & $2(12.5)$ & I (I4.29) & 0.9096 \\
\hline
\end{tabular}

Regardless of growing rates of macrolide resistance to $S$. pneumoniae, the present guidelines for outpatient treatment of CAP include a macrolide as the first empiric therapy. ${ }^{9}$ There have been few formerly documented cases of macrolide treatment failure in CAP, and there is disagreement as to whether macrolide resistance in S. pneumoniae is clinically essential. ${ }^{9}$

The rising reports of clinical treatment failure with macrolides and their connotations with macrolide-resistant $S$. pneumoniae were shown in a review of 33 cases describing macrolide treatment failure in treatment of pneumococcal infections. ${ }^{10}$ The most widespread diagnosis was CAP. Former medical history incorporated cardiopulmonary disease in eight patients and immunocompromised conditions in five patients. The largest percentage of patients obtained oral macrolide treatment in an outpatient setting. S. pneumoniae was separated from the blood in $78.8 \%$ of patients, $9.1 \%$ of patients had bacteria present in both blood and cerebrospinal fluid, $6 \%$ of patients showed S. pneumoniae from blood and bronchial lavage, and $6 \%$ of patients had positive sputum cultures. ${ }^{10}$ In our study, $21.75 \%$ of the cases showed macrolide failure following its use for treatment of CAP caused by S. pneumoniae. S. pneumoniae was isolated in $52.17 \%$ of the cases from the blood culture, $30.43 \%$ of the cases through pulmonary aspiration by bronchoscopy, and from sputum culture in only $4.35 \%$ of the cases.

S. pneumoniae is still the main bacterial cause of CAP and is associated with significant morbidity and mortality worldwide. A key problem is the growing prevalence of antibiotic resistance among pneumococcal isolates, which has been connected with treatment failure. Until now, despite numerous articles about infections with penicillin-resistant pneumococcal isolates, no cases of bacteriologic failure have been reported in the treatment of CAP caused by penicillin-resistant pneumococci by penicillin or ampicillin. ${ }^{11}$ The present incidence rates and levels of penicillin resistance among pneumococcal isolates in most regions of the world do not require substantial treatment changes with respect to the application of penicillin. For infections with penicillin-sensitive strains, penicillin or aminopenicillin in the usual dosage will still be successful for treatment. For cases of strains with intermediate resistance, beta-lactam agents are still believed to be suitable treatments, although higher dosages are recommended. ${ }^{11}$ Infections with isolates of high-level penicillin resistance should be treated with alternatives

Table 4 PCR for detecting the pneumococcal antigen in pulmonary aspiration as compared to PCR of urine and the rapid pneumococcal test

\begin{tabular}{|c|c|c|c|c|}
\hline & $\begin{array}{l}\text { PCR of pulmonary aspiration } \\
(n=4)(\%)\end{array}$ & $\begin{array}{l}\text { PCR of urine } \\
(n=14)(\%)\end{array}$ & $\begin{array}{l}\text { Rapid pneumococcal } \\
\text { test }(n=2)(\%)\end{array}$ & $P$-value \\
\hline Male & $4(100)$ & $8(57.14)$ & I (50) & 0.32853 \\
\hline Female & 0 & $6(42.86)$ & $\mathrm{I}(50)$ & 0.32853 \\
\hline Mean age \pm SD & $5 I \pm 12.4 \mid$ & $60.36 \pm 13.26$ & $64.5 \pm 21.92$ & 0.425083 \\
\hline $\mathrm{CRP}(<6 \mathrm{mg} / \mathrm{L})$ mean $\pm \mathrm{SD}$ & $54.79 \pm 21.01$ & $194.05 \pm \mid 43.42$ & $195.55 \pm 244.73$ & 0.228916 \\
\hline Leukocyte count $(4,000-10,000 / \mu \mathrm{L})$ mean $\pm \mathrm{SD}$ & $12,025 \pm 7,410.07$ & $|5,47| .43 \pm 9,343.98$ & $18,600 \pm 17,394.83$ & 0.716501 \\
\hline Length of hospital stay in days mean \pm SD & $51 \pm|2.4|$ & $60.36 \pm 13.26$ & $64.5 \pm 21.92$ & 0.425083 \\
\hline
\end{tabular}

Abbreviations: PCR, polymerase chain reaction; CRP, C-reactive protein; SD, standard deviation. 
Table 5 Comparison of comorbidities in the PCRs of aspiration and in urine, and the rapid pneumococcal test for identifying the pneumococcal antigen

\begin{tabular}{|c|c|c|c|c|}
\hline & $\begin{array}{l}\text { PCR of pulmonary } \\
\text { aspiration }(n=4)(\%)\end{array}$ & $\begin{array}{l}\text { PCR of urine } \\
(n=14)(\%)\end{array}$ & $\begin{array}{l}\text { Rapid pneumococcal } \\
\text { test }(n=2)(\%)\end{array}$ & $P$-value \\
\hline \multicolumn{5}{|l|}{ Cardiovascular disease } \\
\hline Anemia & 0 & $2(14.29)$ & 0 & 1.0 \\
\hline Atrial tachyarrhythmia & 0 & I (7.|4) & 0 & 1.0 \\
\hline Atrial fibrillation & 0 & $2(14.29)$ & I (50) & 0.3614035 \\
\hline Coronary artery disease & 0 & $2(14.29)$ & 0 & 1.0 \\
\hline Electrolyte disturbance & 0 & I (7.14) & 0 & 1.0 \\
\hline Heparin-induced thrombocytopenia & 0 & I (7.|4) & 0 & 1.0 \\
\hline Hypertension & 0 & $4(28.57)$ & I (50) & 0.283927 \\
\hline Myocardial infarction & 0 & I (7.14) & 0 & 1.0 \\
\hline Sepsis & 0 & $7(50)$ & I (50) & 0.175851 \\
\hline Shock & 0 & I (7.14) & 0 & 1.0 \\
\hline Thrombosis & 0 & I (7.14) & 0 & 1.0 \\
\hline Valvular heart disease & 0 & I (7.14) & 0 & 1.0 \\
\hline \multicolumn{5}{|l|}{ Pulmonary disease } \\
\hline Asbestosis & $\mathrm{I}(25)$ & 0 & 0 & 0.299999 \\
\hline Acute respiratory distress syndrome & 0 & $2(14.29)$ & $\mathrm{I}(50)$ & 0.3614035 \\
\hline Asthma & 0 & I (7.14) & 0 & 1.0 \\
\hline Bronchiectasis & $\mathrm{I}(25)$ & 0 & 0 & 0.299999 \\
\hline Chronic obstructive bronchitis & $\mathrm{I}(25)$ & $7(50)$ & $\mathrm{I}(50)$ & 1.0 \\
\hline Emphysema & 0 & I (7.|4) & 0 & 1.0 \\
\hline Lung tumor & 0 & I (7.14) & 0 & 1.0 \\
\hline Pleural effusion & 0 & I (7.I4) & 0 & 1.0 \\
\hline Pneumothorax & 0 & $2(14.29)$ & 0 & 1.0 \\
\hline Pulmonary cachexia & 0 & $2(14.29)$ & 0 & 1.0 \\
\hline Pulmonary embolism & 0 & $2(14.29)$ & 0 & 1.0 \\
\hline Pulmonary fibrosis & 0 & I (7.14) & 0 & 1.0 \\
\hline Respiratory failure & $2(50)$ & I (7.14) & 0 & 0.103509 \\
\hline Respiratory global insufficiency & 0 & $2(14.29)$ & 0 & 1.0 \\
\hline State after pulmonary embolism & 0 & I (7.14) & 0 & 1.0 \\
\hline State post-tuberculosis & 0 & I (7.14) & 0 & 1.0 \\
\hline \multicolumn{5}{|l|}{ Gastrointestinal disease } \\
\hline Ascites & 0 & I (7.I4) & 0 & 1.0 \\
\hline Appendectomy & $\mathrm{I}(25)$ & $2(14.29)$ & 0 & 0.680702 \\
\hline Gastrointestinal bleeding & 0 & 0 & I (50) & 0.099999 \\
\hline Hepatitis A & 0 & I (7.14) & 0 & 1.0 \\
\hline Hepatitis C & $\mathrm{I}(25)$ & 0 & 0 & 0.299999 \\
\hline Hypercholesterolemia & 0 & I (7.|4) & 0 & 1.0 \\
\hline Liver cirrhosis & $\mathrm{I}(25)$ & 0 & 0 & 0.299999 \\
\hline \multicolumn{5}{|l|}{ Thyroid disease } \\
\hline Hyperthyroidism & 0 & I (7.14) & 0 & 1.0 \\
\hline Hypothyroidism & 0 & I (7.14) & $\mathrm{I}(50)$ & 0.226316 \\
\hline \multicolumn{5}{|l|}{ Kidney and urinary tract diseases } \\
\hline Acute kidney injury & 0 & $2(14.29)$ & 0 & 1.0 \\
\hline Benign prostatic hyperplasia & 0 & I (7.14) & 0 & 1.0 \\
\hline Macrohematuria & 0 & I (7.14) & 0 & 1.0 \\
\hline Urinary retention & 0 & I (7.14) & 0 & 1.0 \\
\hline \multicolumn{5}{|l|}{ Gynecological disorders } \\
\hline Hysterectomy & 0 & I (7.14) & 0 & 1.0 \\
\hline \multicolumn{5}{|l|}{ Orthopedic disorders } \\
\hline Osteoarthritis & 0 & I (7.14) & 0 & 1.0 \\
\hline \multicolumn{5}{|l|}{ Nervous system disorders } \\
\hline Epilepsy & 0 & I (7.| 4$)$ & 0 & 1.0 \\
\hline Prior stroke & 0 & I (7.|14) & 0 & 1.0 \\
\hline \multicolumn{5}{|l|}{ Psychiatric disorders } \\
\hline Alcohol abuse & $\mathrm{I}(25)$ & $2(14.29)$ & 0 & 0.680702 \\
\hline Drug abuse & $\mathrm{I}(25)$ & I (7.14) & 0 & 0.521053 \\
\hline
\end{tabular}


Table 5 (Continued)

\begin{tabular}{|c|c|c|c|c|}
\hline & $\begin{array}{l}\text { PCR of pulmonary } \\
\text { aspiration }(n=4)(\%)\end{array}$ & $\begin{array}{l}\text { PCR of urine } \\
(n=14)(\%)\end{array}$ & $\begin{array}{l}\text { Rapid pneumococcal } \\
\text { test }(n=2)(\%)\end{array}$ & $P$-value \\
\hline Ex-nicotine abuse & 0 & $4(28.57)$ & I (50) & 0.283927 \\
\hline Nicotine abuse & I (25) & $2(14.29)$ & 0 & 0.680702 \\
\hline \multicolumn{5}{|l|}{ Skin disorders } \\
\hline Allergy & 0 & I (7.|4) & I (50) & 0.226316 \\
\hline Exsiccosis & 0 & $2(14.29)$ & 0 & 1.0 \\
\hline
\end{tabular}

Abbreviation: $\mathrm{PCR}$, polymerase chain reaction.

such as the third-generation cephalosporins or the new antipneumococcal fluoroquinolones. ${ }^{11}$ Numerous cases of macrolide azalide treatment failure have been documented, and many physicians have mentioned that these antibiotics not be used on their own in regions with a high incidence and levels of macrolide azalide resistance. ${ }^{11-13}$ However, combination therapy has been shown to be helpful in the most severe cases of patients hospitalized with pneumococcal pneumonia, particularly the combination of a beta-lactam agent and a macrolide. In an effort to avoid resistance, it has been suggested that the new fluoroquinolones not be used routinely as first-line agents in the treatment of CAP. Instead, these agents should be preserved for patients who are allergic to the commonly used beta-lactam agents, for infections known to be or assumed to be triggered by highly resistant strains and for patients for whom initial therapy has been unsuccessful. ${ }^{11}$ Also in this study, azalide, as the active substance in azithromycin, had a statistically significant rate of resistance against $S$. pneumoniae. This medication was not used on the patients observed in this study. Cephalosporins had an above-average effect and no resistance against $S$. pneumoniae in this study. Therefore, according to the data, the antibiotics in this class may be recommended for drug therapy of CAP by S. pneumoniae.
Another study reported that penicillin and macrolide resistance is increasing in Italy and that fluoroquinolone currently continues to be effective. ${ }^{14}$ Another study confirmed the trend in Italy since 1992 of increasing macrolide resistance among respiratory microorganisms - numerous antibiotics, including amoxicillin and clavulanic acid, third-generation injectable cephalosporins, and fluoroquinolones, now have a reduced effect on pathogens, despite large doses. ${ }^{15}$ This current study confirms the effectiveness of these antibiotics on amoxicillin and clavulanic acid but not for fluoroquinolones. Levofloxacin had a high resistance rate in this study.

While penicillin and erythromycin resistance was related to a lower mortality, an association between levofloxacin resistance and an elevated mortality rate was found in the outcome of another investigation. ${ }^{15}$

The failure of levofloxacin treatment in patients with CAP was also reported. ${ }^{16}$ The authors of this study isolated levofloxacin-resistant $S$. pneumoniae in the pleural effusion of two patients. Both patients who had not received previous treatment with quinolones were admitted to the hospital for CAP and initially treated with levofloxacin. The outcome was favorable following chest tube placement and treatment with beta-lactam antibiotics. ${ }^{16}$ The authors of this study proposed that patients with CAP who have obtained quinolones in the

Table 6 Comparison of antibiotics of first choice in the treatment of Streptococcus pneumoniae with change of antibiotics used in resistant germs

\begin{tabular}{|c|c|c|c|}
\hline \multirow[t]{2}{*}{ Drug groups } & \multirow[t]{2}{*}{ Active substance } & \multicolumn{2}{|l|}{$\mathbf{N}=\mathbf{2 3}$} \\
\hline & & $\begin{array}{l}\text { Number of antibiotics } \\
\text { of first choice (\%) }\end{array}$ & $\begin{array}{l}\text { Number of antibiotics } \\
\text { used in resistant germs (\%) }\end{array}$ \\
\hline Penicillin & Penicillin G & I (4.35) & I (4.35) \\
\hline \multirow[t]{2}{*}{ Penicillin + Beta-lactamase inhibitors } & Ampicillin + Sulbactam & $4(17.39)$ & 0 \\
\hline & Piperacillin + Tazobactam & II (47.83) & 0 \\
\hline \multirow[t]{2}{*}{ Cephalosporins } & Ceftazidime & $2(8.70)$ & 0 \\
\hline & Ceftriaxone & $3(13.04)$ & 0 \\
\hline Macrolides & Clarithromycin & $7(30.43)$ & 0 \\
\hline \multirow[t]{2}{*}{ Gyrase inhibitors } & Ciprofloxacin & $2(8.70)$ & 0 \\
\hline & Levofloxacin & $2(8.70)$ & I (4.35) \\
\hline Glycopeptides & Vancomycin & I (4.35) & 0 \\
\hline Carbapenems & Meropenem & 0 & $2(8.70)$ \\
\hline
\end{tabular}


weeks before the occurrence of CAP should not be treated empirically with these drugs because of the risk of developing resistance.

Susceptibility testing for $S$. pneumoniae isolates was performed for eight European countries in another study. ${ }^{17}$ Generally, $24.6 \%$ of $S$. pneumoniae isolates were nonsusceptible to penicillin $\mathrm{G}$, and $28.0 \%$ were resistant to macrolides. The prevalence of resistance varied between European countries, with the highest rates of penicillin $G$ and macrolide resistance being reported in Spain and France. Serotype 14 was the leading serotype among penicillin G and macrolide-resistant $S$. pneumoniae. Fluoroquinolone resistance rates were low (Austria, 0\%; Belgium, $0.7 \%$; France, $0.9 \%$; Germany, $0.4 \%$; Italy, $1.3 \%$; Portugal, $1.2 \%$; Spain, $1.0 \%$; and Switzerland, $0 \%$ ). The analysis of quinolone resistance determined eight strains and showed that the 18 fluoroquinolone-resistant strains were genetically heterogeneous. ${ }^{17}$ Further investigation into the spread of these antibiotic-resistant S. pneumoniae clones is warranted. As shown in the above study, S. pneumoniae has resistance to antibiotics despite significant geographical differences.

To avoid opportunistic bacterial infections and Pneumocystis jirovecii, the treatment proposed by the World Health Organization is a combination of trimethoprim and sulfamethoxazole (co-trimoxazole) for prophylaxis in patients with human immunodeficiency virus and acquired immunodeficiency syndrome. ${ }^{18}$ Although co-trimoxazole does not belong to the first-line treatment of CAP caused by $S$. pneumoniae, resistance to co-trimoxazole is well known. Isolates from patients with CAP caused by $S$. pneumoniae were shown in another study to have $7 \%$ resistance to penicillin, and isolates from $26 \%$ were resistant to co-trimoxazole. ${ }^{19}$ In this study, the resistance rate to co-trimoxazole was $13.04 \%$.

Another study analyzed the case records of cultures for proven pneumococcal infections over a period of 7 years at a rural tertiary health care center and studied the antibiotic resistance of the isolates..$^{20}$ During the study period, pneumococci were separated from 34 samples. All of the isolates were sensitive to penicillin; they were also sensitive to erythromycin, chloramphenicol, vancomycin, and linezolid. The sensitivity to gentamicin, ciprofloxacin, and tetracycline varied from $79.4 \%$ to $88.2 \%$. Only $50 \%$ of the isolates were sensitive to co-trimoxazole. ${ }^{20}$

In this present study, a high resistance rate was detected for erythromycin (17.39\%) and chloramphenicol $(4.35 \%)$. The sensitivity was $17.39 \%$ for gentamicin and $4.35 \%$ for ciprofloxacin. Tetracycline was found to have no sensitivity, with a resistance rate of $4.35 \%$, which is not statistically significant.

Another study examined the clinical effectiveness of the PCR method applied to sputum samples to detect $S$. pneumoniae in patients admitted to hospital with CAP. Induced sputum samples were examined by cultures and PCR. ${ }^{21}$ The pneumococcal antigen was identified in $20 \%$ of the urine samples. In $15 \%$ of the sputum samples, the culture was significantly positive. ${ }^{21}$ In this investigation, the pneumococcal antigen was detected in $70 \%$ of the urine samples and in $20 \%$ of the pulmonary aspiration samples taken by bronchoscopy. S. pneumoniae may be rapidly detected by examining induced sputum samples by PCR, a technique that is most often used for patients in whom antibiotic therapy has already been started.

\section{Study limitations}

This study examined the microbiological growth of microorganisms in patients with CAP in one department of internal medicine but did not investigate patients with CAP in other medical departments. The present study examined a small number of patients with CAP caused by $S$. pneumoniae, so its conclusions may not be extrapolated to other geographic regions.

\section{Conclusion}

This investigation found a high sensitivity to penicillin $G$ and vancomycin after cultures of $S$. pneumoniae were taken from patients with CAP. A resistance profile was detected for the macrolides erythromycin, tetracycline, chloramphenicol, levofloxacin, gentamicin, clindamycin, ampicillin, penicillin $\mathrm{G}$, and co-trimoxazole.

\section{Disclosure}

The author reports no conflict of interest in this work.

\section{References}

1. British Thoracic Society Standards of Care Committee. BTS guidelines for the management of community acquired pneumonia in adults. Thorax. 2001;56(4):1-64.

2. Scott SS, Kardos CB. Community-acquired, health care-associated, and ventilar-associated pneumonia: three variations of a serious disease. Crit Care Nurs Clin North Am. 2012;24:431-441.

3. Kleinpell RM, Elpern EH. Community-acquired pneumonia: updates in assessment and management. Crit Care Nurs Q. 2004;27:231-240.

4. Beekmann SE, Heilmann KP, Richter SS, et al. Antimicrobial resistance in Streptococcus pneumoniae, Haemophilus influenzae, Moraxella catarrhalis and group A beta-haemolytic streptococci in 2002-2003. Results of the multinational GRASP Surveillance Program. Int $J$ Antimicrob Agents. 2005;25:148-156.

5. Kattan R, Abu Rayyan A, Zheiman I, et al. Serotype distribution and drug resistance in Streptococcus pneumoniae. Emerg Infect Dis. 2011;17: 94-96. 
6. International Organization for Standards. Susceptibility testing of infectious agents and evaluation of performance of antimicrobial susceptibility testing devices. 1 . Reference method for testing the in vitro activity of antimicrobial agents against rapidly growing anaerobic bacteria involved in infectious diseases. ISO 20776-1. Geneva, Switzerland: International Organization for Standards; 2006. Available from: http://www.iso.org/iso/iso_catalogue/catalogue_tc/catalogue_detail. htm?csnumber=41630. Accessed July 17, 2014.

7. Imähl M, van der Linden M. [Invasive pneumococcal disease in Germany in the era of pneumococcal conjugate vaccination]. Dtsch Med Wochenschr. 2014;139:1346-1351. German.

8. National Reference Center for Streptococci, Institute of Medical Microbiology, University Hospital RWTH Aachen. 2012. Available from: http://www.pneumococcus.de/. Accessed July 17, 2014.

9. Dylewski J, Davidson R. Bacteremic pneumococcal pneumonia associated with macrolide failure. Eur J Clin Microbiol Infect Dis. 2006; 25:39-42.

10. Rzeszutek M, Wierzbowski A, Hoban DJ, Conly J, Bishai W, Zhanel GG. A review of clinical failures associated with macrolide-resistant Streptococcus pneumoniae. Int J Antimicrob Agents. 2004;24:95-104.

11. Aspa J, Rajas O, de Castro FR. Pneumococcal antimicrobial resistance: therapeutic strategy and management in community-acquired pneumonia. Expert Opin Pharmacother. 2008;9:229-241.

12. Metzler K, Drilica K, Blondeau JM. Minimal inhibitory and mutant prevention concentrations of azithromycin, clarithromycin and erythromycin for clinical isolates of Streptococcus pneumoniae. J Antimicrob Chemother. 2013;68:631-635.

13. Schito GC, Felmingham D. Susceptibility of Streptococcus pneumoniae to penicillin, azithromycin and telithromycin (PROTEKT 1999-2003). Int J Antimicrob Agents. 2005;26:479-485.

14. Marchese A, Gualco L, Cochetti I, et al. Antibiotic susceptibility and serotype distribution in Streptococcus pneumoniae circulating in Italy: results of the SEMPRE surveillance study (2000-2002). Int J Antimicrob Agents. 2005;26:138-145.
15. Kang CI, Song JH, Kim SH, et al. Asian Network for Surveillance of Resistant Pathogens (ANSORP) Study Group. Association of levofloxacin resistance with mortality in adult patients with invasive pneumococcal diseases: a post hoc analysis of a prospective cohort. Infection. 2013;41:151-157.

16. Carlavilla AB, López-Medrano F, Chaves F, Villena V, Echave-Sustaeta J, Aguado JM. [Failure of levofloxacin therapy in two cases of communityacquired pneumonia caused by fluoroquinolone-resistant Streptococcus pneumoniae and complicated with empyema]. Enferm Infecc Microbiol Clin. 2005;23:270-273. Spanish.

17. Reinert RR, Reinert S, van der Linden M, Cil MY, Al-Lahham A, Appelbaum P. Antimicrobial susceptibility of Streptococcus pneumoniae in eight European countries from 2001 to 2003. Antimicrob Agents Chemother. 2005;49:2903-2913.

18. WHO. HIV/AIDS Guidelines. Available from: http://www.who.int/ hiv/pub/guidelines/en/. Accessed July 17, 2014.

19. Hofmann J, Cetron MS, Farley MM, et al. The prevalence of drugresistant Streptococcus pneumoniae in Atlanta. N Engl J Med. 1995;333: $481-486$.

20. Deva A, Prasad SR, Madappa BP, Junjegowda K, Bachu RP. Pneumococcal infections at a rural tertiary care hospital: a seven year study on isolation rate, clinical spectrum and antibiogram. J Clin Diag Res. 2014;8:50-52.

21. Johansson N, Kalin M, Giske CG, Hedlund J. Quantitative detection of Streptococcus pneumoniae from sputum samples with real-time quantitative polymerase chain reaction for etiologic diagnosis of communityacquired pneumonia. Diagn Microbiol Infect Dis. 2008;60:255-261.

\section{Publish your work in this journal}

Drug Design, Development and Therapy is an international, peerreviewed open-access journal that spans the spectrum of drug design and development through to clinical applications. Clinical outcomes, patient safety, and programs for the development and effective, safe, and sustained use of medicines are a feature of the journal, which

\section{Dovepress}

has also been accepted for indexing on PubMed Central. The manuscript management system is completely online and includes a very quick and fair peer-review system, which is all easy to use. Visit http://www.dovepress.com/testimonials.php to read real quotes from published authors.

Submit your manuscript here: http://www.dovepress.com/drug-design-development-and-therapy-journal 